GT2013-95833

\title{
INTEGRATION OF FLUID THERMODYNAMIC AND TRANSPORT PROPERTIES IN CONCEPTUAL TURBOMACHINERY DESIGN
}

\author{
Egill Thorbergsson, Prof. Tomas Grönstedt \\ Chalmers University of Technology \\ Gothenburg, 412 96, Sweden \\ \{egill, tomas.gronstedt\}@chalmers.se
}

\author{
Christopher Robinson \\ PCA Engineers \\ Lincoln LN2 2LL, UK \\ chris.robinson@pcaeng.co.uk
}

\begin{abstract}
The interest in designing gas turbine cycles that use unconventional working fluids is increasing. To be able to design the turbomachinery for these cycles the design programs need to be modified. This paper describes how a streamline curvature compressor design program, a one-dimensional compressor design program and a cycle performance program are linked to a thermodynamic properties program. A case study is described where the streamline curvature program is used to design a three stage compressor that has air as a working fluid. The design is completed both using a semiperfect gas assumption and the new modification using a state of the art real gas data thermodynamic properties program. If not properly implemented real gas data retrieval may completely dominate calculation times. An algorithm targeting high numerical efficiency intended for through-flow multidimensional optimization is outlined. Various implementation recommendations associated with the integration of the real gas data program into the one-dimensional compressor design program and the cycle program are described.
\end{abstract}

\section{NOMENCLATURE}

$$
\begin{aligned}
& \widehat{m}=\frac{\dot{m} \sqrt{\gamma R T_{01}}}{D^{2} p_{01}} \quad \text { Non-dimensional mass flow } \\
& \widehat{N}=\frac{N D}{\sqrt{\gamma R T_{01}}} \quad \text { Non-dimensional rotational speed } \\
& \gamma=\frac{c_{p}}{c_{p}-R} \quad \text { Ratio between specific heats }
\end{aligned}
$$

$\begin{array}{ll}\eta & \text { Polytropic efficiency } \\ R e & \text { Reynolds number } \\ c_{p} & \text { Specific heat at constant pressure }[\mathrm{J} /(\mathrm{kg} \mathrm{K})] \\ R & \text { Gas constant }[\mathrm{J} /(\mathrm{kg} \mathrm{K})] \\ \phi_{n} & \begin{array}{l}\text { Flow coefficient, where } \mathrm{n} \text { is the number of } \\ \text { the stage }\end{array} \\ \psi_{n} & \text { Stage loading, where } \mathrm{n} \text { is the number of the } \\ \dot{m} & \text { stage } \\ N & \text { Mass flow [kg/s] } \\ T_{0} & \text { Rotational speed [rpm] } \\ p_{0} & \text { Stagnation temperature }[\mathrm{K}] \\ D & \text { Stagnation pressure }[\mathrm{kPa}] \\ P R & \text { Inlet shroud diameter }[\mathrm{m}] \\ \mathrm{IGV} & \text { Pressure ratio } \\ \mathrm{R} & \text { Inlet guide vanes } \\ \mathrm{S} & \text { Rotor } \\ \mathrm{S}-\mathrm{P} & \text { Stator } \\ \text { DLL } & \text { Semi-perfect } \\ \text { REF } & \text { Dynamic-link library } \\ \text { REFPROP } & \text { REFPROP } \\ \text { SCOC-CC } & \begin{array}{l}\text { Reference Fluid Thermodynamic and } \\ \text { cycle }\end{array} \\ & \end{array}$




\section{INTRODUCTION}

The interest to use unconventional working media in gas turbines is increasing. Two examples, which are being researched, are the oxy-fuel combustion combined cycle and the supercritical carbon dioxide cycle. The interest in oxy-fuel power cycles is high since the cycles have a possibility to be used in carbon capture and storage schemes. The supercritical $\mathrm{CO}_{2}$ cycles are of interest since research has indicated that the cycles have a possibility to achieve high efficiencies at moderate pressure ratios [1].

Most conceptual design programs for turbomachinery assume that the media is a calorically perfect gas or semiperfect. However, when dealing with working fluids that have, for example, a large fraction of steam, the simplified assumptions start to deteriorate. An example of a gas turbine that has this property is the Graz cycle, which has as high as $70 \%$ of steam in its working media [2].

A method to take into account the thermodynamics of real gas is to use lookup tables that contain the properties of the gas. Boncinelli et al [3] have for example implemented a numerical model for gas properties in a three-dimensional solver for the compressible Reynolds averaged Navier-Stokes equations to account for real gas effects in turbomachinery flows. These was accomplished by using gas property tables and further exploit a local fitting of the gas data to compute the thermodynamic properties in each solution step. The tables are generated offline so the computational time is minimized. The results from the study by Boncinelli et al show that using the method, the computational time was increased by $14 \%$ compared to when perfect gas is assumed. The study concluded that this was though an effective method to take into account real gas effects.

This is similar to what has been done in other codes, e.g. Casey and Robinson [4] used tables containing real gas data to calculate thermodynamic properties in a new streamline curvature throughflow method for radial turbomachinery. Barsi et al [5] also implemented tables with real gas data to compute thermodynamic properties in a throughflow code for evaluating performance of multi-stage turbines. Dubitsky et al [6] have chosen to go a different route their reduced order though-flow code and instead of table implement NASA GASP, ASME steam and D. B. Robinson real-gas libraries into the solver. There is however, no discussion about the computational time associated with the difference in fidelity of the models.

In the current study, a different method is proposed to consider real gas effects. We present number of modification to existing design programs and discuss the modification from an engineering viewpoint. In association with this practical implementation, issues and lessons learned are described. The proposed modifications are based on linking the various design programs to the Reference Fluid Thermodynamic and Transport Properties Database (REFPROP), which is developed, by National Institute of Standards and Technology [7]. To the authors knowledge the wealth of number of species, species mixtures and the accuracy of the modeling makes the REFPROP database unprecedented for real gas modeling. In addition, a license to the code provides the user full access to the source code of the modeling framework. This tool was therefore chosen as the basis for the thermodynamic property calculations. As examples of this modification, we have used the two dimensional compressor design code SC90C, a meanline correlation based conceptual compressor design code and a thermodynamic design and off-design code IPSEpro [8, 9].

The streamline curvature based code is a commercial program that is developed by PCA Engineers [8]. The code is used to design compressors both using the semi-perfect gas properties assumption, a calorically perfect gas assumption and the new modification incorporating a connection to REFPROP. The implementation is illustrated for a given design case demonstrating its practical usage and computational performance implications. An implementation targeting high speed computations needed when streamline curvature codes are connected to external optimizers is outlined. This implementation is similar to using a lookup table for gas properties.

The mean-line code is used to illustrate particular issues encountered when linking the REFPROP library to an in-house conceptual compressor design code developed in MATLAB. It is also discussed how to establish a numerically efficient implementation for this environment. Some brief illustrations of its usage are discussed. Finally, a case for the commercial cycle analysis software, IPSEpro is discussed.

\section{REAL GASES}

One of the examples where there is need to use more advanced thermodynamic properties calculations is the oxy-fuel combustion combined cycles [10]. Oxy-fuel cycles use oxygen instead of air in the combustion of the fuel. This leads to the fact that the combustion products consist mainly of $\mathrm{CO}_{2}$ and $\mathrm{H}_{2} \mathrm{O}$. Two cycles that are being researched and show good potential are the semi-closed oxy-fuel combustion combined cycle (SCOC-CC) [11] and the Graz cycle [10]. The compositions of the working fluid of the gas turbine for the two cycles are shown in Table 1. The compositions are quite different; the SCOC-CC has a very high content of $\mathrm{CO}_{2}$ while the Graz cycle has a high content of steam. Another aspect of the cycles is that the amount of argon in the working fluid can be considerable. This needs to be taken into account when thermodynamic properties are computed in both the cycle 
simulations and during the conceptual design of the turbomachinery.

It has been shown that these cycles need high pressure ratios to achieve high efficiencies $[2,11]$. The specific heat at constant pressure for the working fluids in each cycle and for air is shown as function of temperature at 1 bar and 40 bars in Figure 1. In Figure 1, it can be seen that for working fluids that have a high content of $\mathrm{H}_{2} \mathrm{O}$ and $\mathrm{CO}_{2}$ real gas effects need to be taken into account at high pressure levels.

TABLE 1. COMPOSITION AND THE GAS CONSTANT FOR THE WORKING MEDIA IN THE SCOC-CC, THE GRAZ CYCLE AND FOR AIR.

\begin{tabular}{|c|c|c|c|c|c|c|}
\hline & \multicolumn{5}{|c|}{ Composition [Mass \%] } & \multirow{2}{*}{$\begin{array}{c}\mathrm{R} \\
{[\mathrm{J} /(\mathrm{kg} \mathrm{K})]}\end{array}$} \\
\hline & $\mathrm{Ar}$ & $\mathrm{CO}_{2}$ & $\mathrm{H}_{2} \mathrm{O}$ & $\mathrm{N}_{2}$ & $\mathrm{O}_{2}$ & \\
\hline Dry Air & 1.27 & 0.00 & 0.00 & 75.6 & 23.2 & 287 \\
\hline SCOC-CC & 4.06 & 92.0 & 0.98 & 2.86 & 0.12 & 196 \\
\hline Graz & 1.55 & 20.9 & 77.4 & 0.03 & 0.13 & 400 \\
\hline
\end{tabular}

\section{MODELLING REAL GAS EFFECTS IN CONCEPTUAL TURBOMACHINERY DESIGN}

REFPROP is based on highly accurate models that are used to calculate the thermodynamic properties of pure fluids and mixtures. NIST states that: "These equations are the most accurate equations available world wide" [12]. To calculate the thermodynamic properties of pure fluids the program uses three models: equations of state explicit in Helmholtz energy, the modified Benedict-Webb-Rubin equations of state, and an extended corresponding states model. Calculations for mixtures use a model that applies mixing rules to the Helmholtz energy of the mixture components. To account for the departure from ideal mixing it uses a departure function [7].

\section{The streamline curvature design program}

The streamline curvature approach solves the governing equations in the meridional plane. The streamline curvature method is based on iterations of the locations of the streamlines using the radial equilibrium and continuity equations, as illustrated in Figure 2. The assumptions made when using the streamline curvature approach are that the flow is steady, adiabatic, axisymmetric, inviscid, and with negligible body forces. More details and discussions regarding the streamline curvature method can be found in Cumpsty [13] and Schobeiri [14].

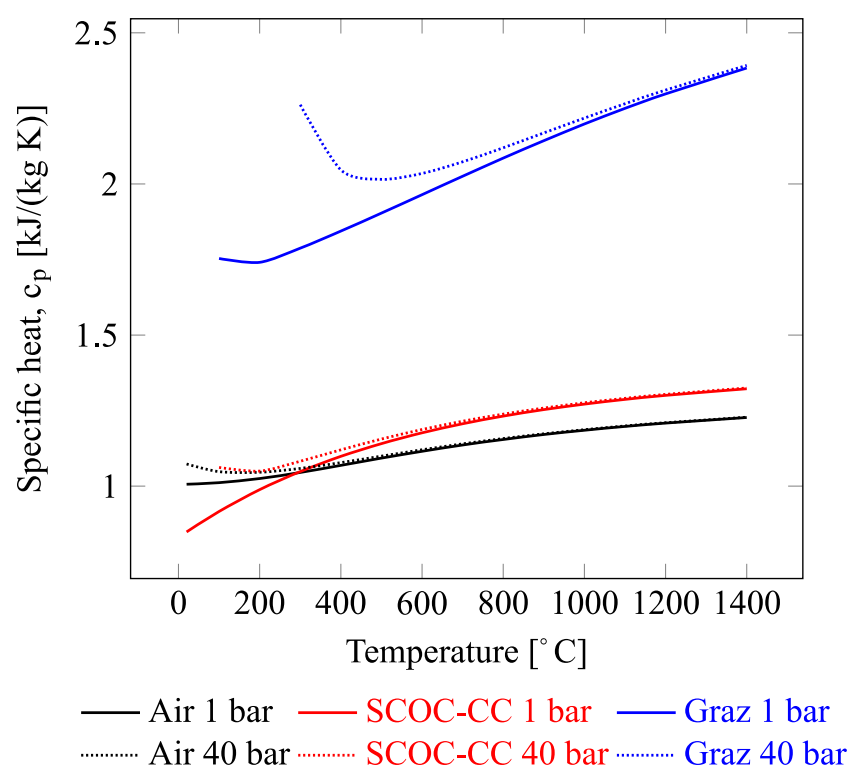

FIGURE 1. SPECIFIC HEAT FOR AIR, THE WORKING FLUID IN THE SCOC-CC, AND THE GRAZ CYCLE, AT 1 BAR AND 40 BAR.

The code uses the loss model and calculation for annulus wall boundary layer developed by Wright and Miller [15]. The code also incorporates the spanwise mixing scheme from Gallimore [16]. The throughflow code uses a semi-perfect gas assumption when the working fluid is dry air. This means that the program uses a polynomial function of temperature to calculate the specific heat. When the working fluid is not dry air, the program assumes a calorically perfect gas, which means that the specific heats ratio is assumed constant. The gas constant and the specific heats ratio are then input variables.

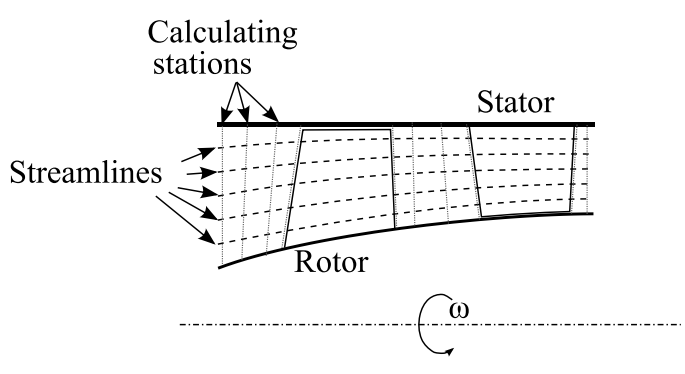

FIGURE 2. STREAMLINE CURVATURES IN A COMPRESSORS STAGE (MERIDIONAL PLANE). 


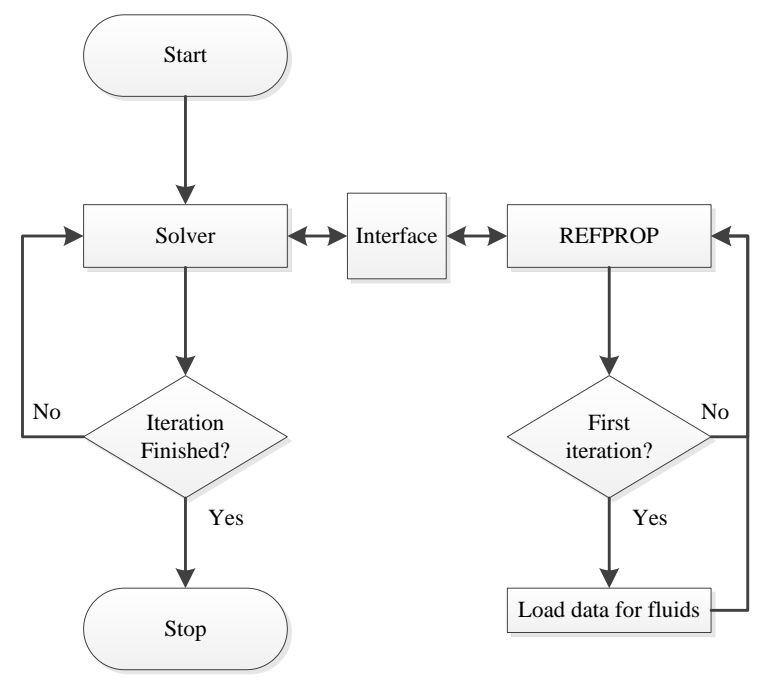

FIGURE 3. FLOW CHART FOR THE MODIFICATIONS OF THE STREAMLINE CURVATURE PROGRAM.

The linking from the SC90C to REFPROP is done using the FORTRAN source codes for both programs. The implementation is shown in Figure 3. A modification has been implemented in the REFPROP code so that the data for each fluid component is loaded only in the first iteration. This is accomplished by employing a common variable, which is zero before the first iteration but has a non-zero value during subsequent iterations. This decreases the computational time by approximately a factor of three. Still, the computational time for the streamline curvature program when using REFPROP, compared to using the standard method, was seen to increase considerably and is now counted in minutes instead of seconds. The order of magnitude for calculation times are increased approximately by a factor of 100 . This is considered to be acceptable for normal design work but needs further improvement when the throughflow code is part of an optimization environment. A solution to improve the calculation performance for such an integrated environment is discussed below.

\section{One-dimensional meanline design program}

A Chalmers in-house meanline compressor design code has been linked to the REFPROP thermodynamic properties program. The code is implemented using MATLAB and is using empirical relations to estimate the losses that are generated. The profile and endwall loss models are based on the work by Wright and Miller [15]. A more detailed discussion of the compressor code algorithms and loss model implementation is presented in [10].
One of the drawbacks of linking MATLAB programs to exterior functions through dynamically-linked subroutines is that the exterior functions are initialized for each call. Since the REFPROP program initializes by reading data for each fluid in the mixture, this increases the computational time considerably. Consequently, this makes the numerical performance of optimization studies completely dominated by the time needed to execute the real gas data modeling software. Since this limitation is due to a built-in MATLAB solution, associated with how MATLAB communicates with external DDL:s, the developer has to resort to other solutions. The most convenient alternative for this particular instance is to generate tables or interpolation functions for every new species mixture that is needed. This can also be overcome by using the same procedure as is used in the implementation for throughflow optimization described below.

\section{Cycle simulation program}

The cycle simulation tool IPSEpro has also been linked to the REFPROP code. IPSEpro is a heat and mass balance program, which was initially developed by Perz [17, 18, 19] and is today developed by SimTech Simulation Technology [9]. The program uses a damped Newton-Raphson based strategy to solve the equation systems that represent the cycles. A DLL was constructed that implemented the thermodynamics functions needed in IPSEpro. In this instance, the DLL can be made to initialize only in the first call in the iteration. This is achieved by the same procedure as discussed above for the streamline curvature design program. The computational time is still longer than when assuming ideal gas and is measured in minutes rather than seconds.

A side effect of the implementation of a REFPROP interface to IPSEpro is that it removes a built-in limitation in the original IPSEpro release. IPSEpro can only handle ideal gas modeling and is therefore limited to analysis up to 35 bars pressure [9]. With the REFPROP interface, this limitation can be removed.

\section{Implementation for throughflow optimization}

When throughflow design programs are used for multidimensional and multi-objective optimization, the computational time starts to play an important role. Multiobjective optimization is normally combined with multidimensional optimization, requiring that a large number of designs be evaluated against several criteria such as component efficiency and aerodynamic stability [20]. A new method to link REFPROP to the design programs is therefore proposed. The implementation will decrease the computational time drastically. The method is to either use the semi-perfect gas 
assumption by employing polynomials that are functions of temperature, or extended polynomials incorporating pressure dependency, to calculate the thermodynamic properties. The polynomials would be generated only in the initialization phase of the optimization using REFPROP to cover a sufficiently large interpolation range. In a practical optimization scenario the user will usually have a good understanding of the search space in terms what is the expected minimum and maximum pressures and temperatures. This information then has to be passed to the REFPROP initialization process. Without such information the interface can assume a large parameter range that possibly will have to be extended through a reinitialization, if the optimizer will go into a completely unexpected region.

A flow chart of the method adapted for optimization is shown in Figure 4. The polynomials can normally be generated one time for each gas mixture and then be used in multiple runs within the throughflow design code. The suggested algorithm will also minimize the amount of modification needed for the through flow code. Since the polynomial generation can be automated, all fluids and fluid mixtures available in REFPROP, a library that is constantly growing, are thus made accessible to the user. It should be pointed out that this implementation is believed to be easily extended to any throughflow code implementation for which the source code is available.

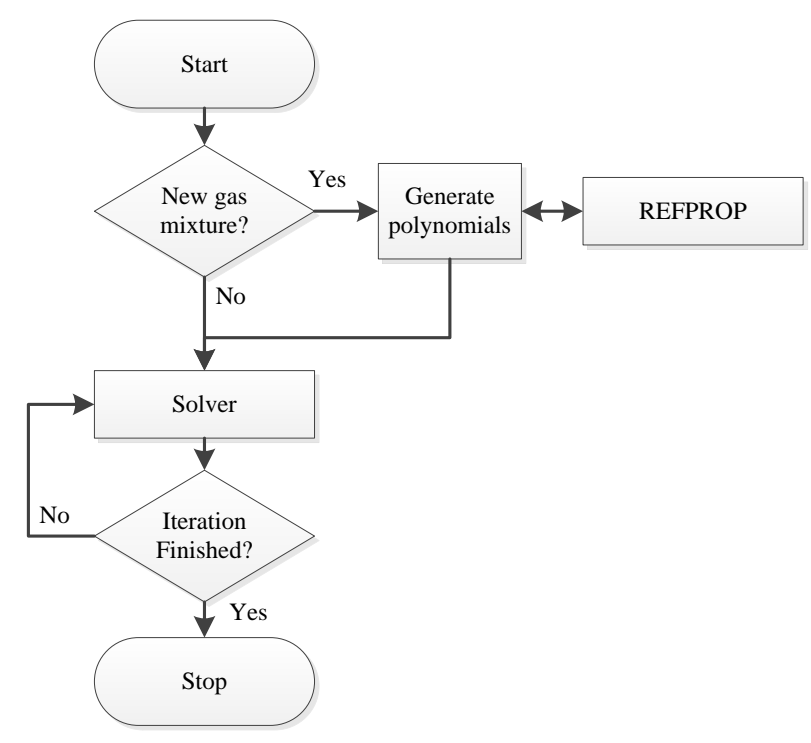

FIGURE 4. FLOW CHART FOR THE IMPLEMENTATION ADAPTED FOR OPTIMIZATION.

The optimization adapted procedure can be implemented both in the streamline curvature program and the meanline design program. The method is however not possible to implement in the cycle simulation tool since the working fluid mixture is not the same at each stream in the cycle and can change between each iteration.

\section{CASE STUDIES}

A case study has been done using the streamline curvature program. The purpose is to validate the modification of the compressor design code, i.e. to ensure that the interface reproduces the expected results. For an estimate of the deviations caused by real gas effects the user is referred to Figure 1, as well as an additional study presented below.

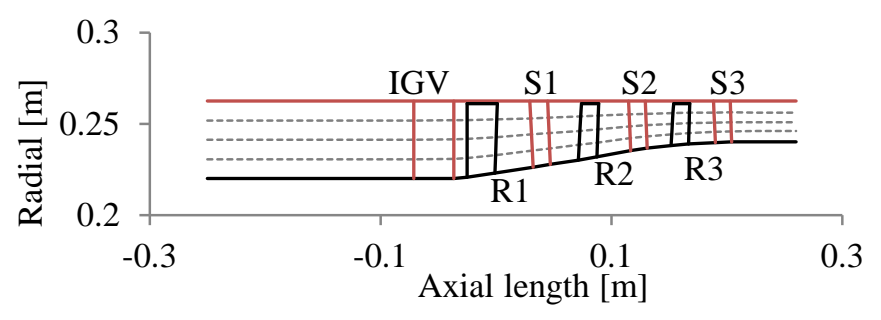

FIGURE 5. A MERIDIONAL VIEW OF THE COMPRESSOR

The design case is a three stage compressor. The meridional view of the compressor is shown in Figure 5. The design parameters for the compressor are

- Mass flow: $\dot{m}_{\text {des }}=10.65 \mathrm{~kg} / \mathrm{s}$

- Rotation speed: $N_{\text {des }}=9300 \mathrm{rpm}$

- Inlet pressure: $p_{01}=101.325 \mathrm{kPa}$

- Inlet temperature: $T_{01}=288.15 \mathrm{~K}$

The working fluid used when originally designing the compressor was dry air. The streamline curvature code is here used in the design mode. The thermodynamic properties are computed both using the semi-perfect assumption and the REFPROP modification. The resulting efficiency, pressure ratio, flow coefficient, and stage loading for both cases are shown in Table 2. The results for the two methods are in good agreement. 
TABLE 2. SIMULATION RESULTS FOR THE THREESTAGE COMPRESSOR.

\begin{tabular}{ccccccccc}
\hline & $P R$ & $\eta[\%]$ & $\phi_{1}$ & $\phi_{2}$ & $\phi_{3}$ & $\psi_{1}$ & $\psi_{2}$ & $\psi_{3}$ \\
\hline S-P & 2.48 & 88.63 & 0.67 & 0.67 & 0.64 & 0.58 & 0.61 & 0.51 \\
Ref & 2.51 & 88.56 & 0.67 & 0.67 & 0.63 & 0.59 & 0.62 & 0.52 \\
\hline
\end{tabular}

The non-dimensional groups for a compressor are

$$
\frac{p_{02}}{p_{01}}, \eta, \frac{\Delta T_{0}}{T_{01}}=f\left\{\frac{\dot{m} \sqrt{\gamma R T_{01}}}{D^{2} p_{01}}, \frac{N D}{\sqrt{\gamma R T_{01}}}, R e, \gamma\right\}
$$

which shows that the efficiency and the pressure ratio are functions of non-dimensional mass flow, non-dimensional rotation speed, the Reynolds number and the specific heat ratio [21]. The pressure ratio variation of the compressor is shown in Figure 6 and the efficiency variations is shown in Figure 7; in both instances as functions of the non-dimensional mass flow and the rotational speed. The performance maps are generated using the semi-perfect gas assumption and that the working fluid is dry air. The maps are also generated using the REFPROP modification and are shown in Figures 6 and 7.

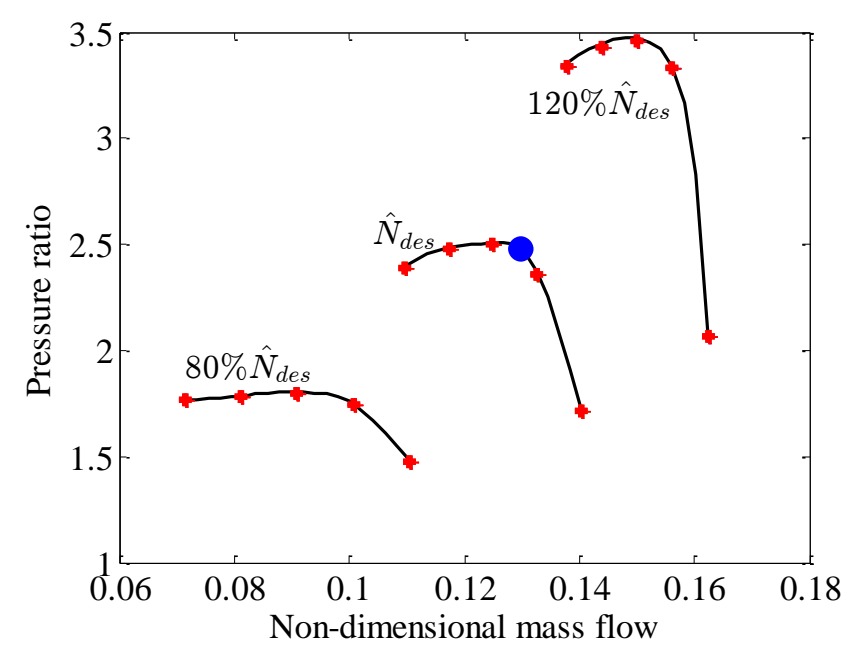

FIGURE 6. COMPRESSOR PRESSURE RATIO VARIATION. THE CIRCULAR DOT INDICATES THE AERODYNAMIC DESIGN POINT. BLACK LINES INDICATE IDEAL GAS AND THE RED DOTS INDICATE REFPROP.

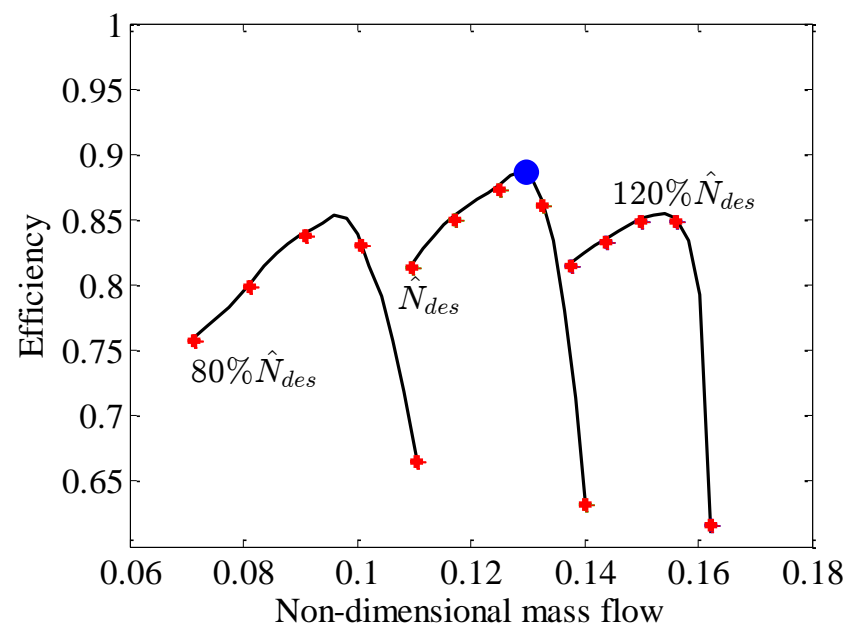

FIGURE 7. EFFICIENCY VARIATION. THE CIRCULAR DOT INDICATES THE AERODYNAMIC DESIGN POINT. BLACK LINES INDICATE IDEAL GAS AND THE RED DOTS INDICATE REFPROP.

The streamline curvature program was then used in analysis mode to examine how the performance of the compressor changes when water is introduced into the working fluid. The design chosen as input for the study was the resulting design using the semi-perfect gas assumption. The amount of water is varied from $0 \%$ to $15 \%$ of the mass flow, in $5 \%$ increments. It is assumed that the water is in the gas phase for all the cases.

The results from the study using the streamline curvature program are shown in Table 3 , both using the calorically perfect gas assumption and using REFPROP to calculate the thermodynamic properties. The reason for using a calorically perfect as a basis for this comparison is that the SC90C program only allows using the semi-perfect gas model for dry air.

As the water content is increased the performance of the compressor deteriorates. This comes from the fact that as the water content increases the gas constant and specific heat ratio will change. The non-dimensional mass flow will therefore also increase while the non-dimensional rotational speed will decrease. This results in that the pressure ratio and the efficiency will drop.

As can be seen in Table 3 the agreement between the calorically perfect gas assumption and the REFPROP method is good. This is to be expected since the pressure level for this validation case was set to be in a region that does not introduce real gas effects, and the temperature range within the threestage compressor is modest. Hence, the effect of a constant specific heat associated with a calorically perfect gas is small. 
TABLE 3. RESULTS FROM THE THROUGHFLOW CODE WITH VARYING THE WATER CONTENT OF THE WORKING FLUID.

\begin{tabular}{lcccc}
\hline Water [\%mass] & $0 \%$ & $5 \%$ & $10 \%$ & $15 \%$ \\
\hline$P R_{\text {ideal }}$ & 2.48 & 2.24 & 1.89 & 1.54 \\
$P R_{\text {refprop }}$ & 2.47 & 2.23 & 1.88 & 1.55 \\
\hline$\eta_{\text {ideal }}$ & $88.63 \%$ & $84.23 \%$ & $73.03 \%$ & $57.22 \%$ \\
$\eta_{\text {refprop }}$ & $88.27 \%$ & $83.86 \%$ & $72.73 \%$ & $57.43 \%$ \\
\hline$R$ & 287 & 296 & 305 & 313 \\
$\gamma$ & 1.40 & 1.40 & 1.39 & 1.39 \\
$\widehat{m}$ & 0.130 & 0.132 & 0.133 & 0.135 \\
$\widehat{N}$ & 14.34 & 14.15 & 13.98 & 13.80 \\
\hline
\end{tabular}

\section{Real gas effects}

It is possible to check how the two methods will behave when the working fluid is in the region where there is a need to consider real gas effects. This is made possible by increasing the inlet temperature and the inlet pressure while keeping the non-dimensional mass flow and the non-dimensional rotational speed constant. The inlet pressure is chosen as $p_{01}=1600 \mathrm{kPa}$ and the inlet temperature is chosen as $T_{01}=450 \mathrm{~K}$. The nondimensional mass flow and rotational speed are chosen to be the same as for the design case, that is $\widehat{m}=0.130$ and $\widehat{N}=14.34$. The results from the streamline curvature program, both using the calorically perfect gas assumption and the REFPROP modification are shown in Table 4. It is seen that the calorically perfect gas assumption over predicts the efficiency and the pressure ratio.

TABLE 4. RESULTS FROM THE THROUGHFLOW CODE WHEN INCREASING THE INLET TEMPERATURE AND PRESSURE.

\begin{tabular}{lc}
\hline Water [\%mass] & $15 \%$ \\
\hline$P R_{\text {ideal }}$ & 2.56 \\
$P R_{\text {refprop }}$ & 2.46 \\
\hline$\eta_{\text {ideal }}$ & $89.76 \%$ \\
$\eta_{\text {refprop }}$ & $86.57 \%$ \\
\hline
\end{tabular}

\section{CONCLUSION AND DISUCSSION}

A number of methods on how to practically include real gas thermodynamic effects into turbomachinery design programs have been presented. As discussed, the need to incorporate detailed real gas fluid modeling exists for instances for gas turbine cycles that have working fluids with a high content of steam designed for high pressure ratios. It is the belief of the authors that the paper provides solutions to research and engineering problems of a currently growing interest. Some particular findings on how to implement numerically efficient solutions for through-flow based optimizers and particular pitfalls for interfacing the REFPROP software to conceptual design tools and cycle analysis tools are provided.

\section{ACKNOWLEDGMENTS}

This research has been funded by the Swedish Energy Agency, Siemens Industrial Turbomachinery AB, Volvo Aero Corporation and the Royal Institute of Technology through the Swedish research program TURBOPOWER. The support of which is gratefully acknowledged.

The authors want to thank Dr. Eric W. Lemmon for providing useful information in regards to the REFPROP code.

\section{REFERENCES}

[1] K. Kimball and E. Clementoni, "Supercritical carbon dioxide brayton power cycle development overview," in ASME Turbo Expo 2012: Power for Land, Sea, and Air, Copenhagen, 2012.

[2] E. Thorbergsson, T. Grönstedt, M. Sammak and M. Genrup, "A comparative analysis of two competing mid-size oxy-fuel combustion cycles," in ASME Turbe Expo 2012: Power for Land, Sea and Air, Copenhagen, 2012.

[3] P. Boncinelli, F. Rubechini, A. Arnone, M. Cecconi and C. Cortese, "Real Gas Effects in Turbomachinery Flows: A Computational Fluid Dynamics Model for Fast Computations," Journal of turbomachinery, vol. 126, pp. 268-276, 2004.

[4] M. Casey and C. Robinson, "A New Streamline Curvature Throughflow Method for Radial Turbomachinery," in Proceedings of ASME Turbo Expo 2008: Power for Land, Sea and Air, Berlin, 2008.

[5] D. Barsi, R. Canepa and A. Satta, "A semi-empirical procedure for the evaluation of multi-stage turbine performances," in International Conference on Renewable Energies and Power Quality, Las Palmas de Gran Canaria, 2011. 
[6] O. Dubitsky, A. Wiedermann, T. Nakano and J. Perera, "The Reduced Order Through-Flow Modeling of Axial Turbomachinery," in Proceedings of the International Gas Turbine Congress, Tokyo, 2003.

[7] E. Lemmon, M. McLinden and M. Huber, NIST Standard Reference Database 23: Reference Fluid Thermodynamic and Transport Properties-REFPROP, Gaithersburg: National Institute of Standards and Technology, 2007.

[8] PCA-Engineers, SC90c - A streamline curvature program for axial compressors, Lincoln, 2008.

[9] SimTech Simulation Technology, User Documentation: Program Modules and Model, IPSEpro Process Simulator, 2003.

[10] E. Thorbergsson, Conceptual Gas Turbine Modelling for Oxy-fuel Power Cycles, Gothenburg: Chalmers University of Technology, 2012.

[11] M. Sammak, Jonshagen, Thern, Genrup, Thorbergsson, Grönstedt and Dahlquist, "Conceptual design of a midsized semi-closed oxy-fuel combustion combined cycle," in ASME Turbo Expo 2011: Power for Land, Sea and Air, Vancouver, 2011.

[12] E. Lemmon, "Answers to Frequently Asked Questions," National Institute of Standards and Technology, Okt 2012. [Online]. Available: http://www.boulder.nist.gov/div838/theory/refprop/Fre quently_asked_questions.htm. [Accessed Okt 2012].

[13] N. A. Cumpsty, Compressor aerodynamics, 2nd ed., New York: Krieger scientific, 2004.

[14] M. Schobeiri, Turbomachinery Flow Physics and Dynamic Performance, Berlin: Springer, 2005.

[15] P. Wright and D. Miller, "An Improved Compressor Performance Prediction Model," IMechE paper C423/028, 1991.

[16] S. Gallimore, "Spanwise Mixing in Multistage Axial Flow Compressors. Part II - Throughflow Calculations Including Mixing," Journal of Turbomachinery, p. 108, 1986.

[17] E. Perz, "A computer method for thermal power cycle calculation," Journal of Engineering for Gas Turbines and Power, vol. 113, no. 2, pp. 184-189, 1991.

[18] E. Perz, "Computer aided analysis of thermal power processes," in ASME Cogen-Turbo Power Conference, Cincinnati, 1993.

[19] D. Fátima and E. W. Perz, "A decision support system for power plant design," European journal of operational research, vol. 109, no. 2, pp. 310-320, 1998.

[20] E. Thorbergsson and T. Grönstedt, "Multicriteria opimization of conceptual compressor aerodynamic design," in International Society for Airbreating Engines, Gothenburg, 2011.
[21] C. Hall and S. Dixon, Fluid Mechanics and Thermodynamics of Turbomachinery, ButterworthHeinemann, 2010.

[22] D. C. Miller and D. L. Wasdell, "Off-Design Prediction of Compressor Blade Losses," IMechE paper C279/87, 1987. 